\title{
Theoretical and experimental studies of the photoemission current from $\mathrm{GaAs}(110)$
}

\author{
J. Henk and W. Schattke \\ Institut für Theoretische Physik und Sternwarte, Christian-Albrechts-Universität, Leibnizstrasse 15, \\ D-2300 Kiel, Federal Republic of Germany
}

H.-P. Barnscheidt, C. Janowitz, R. Manzke, and M. Skibowski

Institut für Experimentalphysik, Christian-Albrechts-Universität, Leibnizstrasse 15, D-2300 Kiel, Federal Republic of Germany

(Received 14 December 1988)

\begin{abstract}
We report calculations of photoemission spectra including final-state effects for normal emission from $\mathrm{GaAs}(110)$ and compare the results with measurements. In the calculations we consider the matrix elements between initial tight-binding and final pseudopotential wave functions. All peaks of the experimental energy-distribution curves can be explained in the direct-transition model using structure plots, except one transition, which is explained by a surface state. Experimental and theoretical spectra compare well concerning energy position and intensity.
\end{abstract}

\section{INTRODUCTION}

Angle-resolved photoemission spectroscopy (ARPES) is an important tool for the experimental determination of semiconductor energy-band structures. With this technique one is able to study bulk as well as surface bands. For instance, the GaAs(110) surface has been the object of several ARPES measurements (see, for example, Refs. 1 and 2), and is at present one of the best experimentally understood semiconductor surfaces. Therefore it lends itself as a prototype for a theoretical photoemission calculation which is closely related to energy-bandstructure calculations including surface bands. There are various theoretical methods for the calculation of energy bands. In the region of the valence bands, empirical tight-binding methods (ETBM's) are used successfully to calculate the band structure of the bulk and the surface. ${ }^{3}$ Because of their simplicity, these methods are still in use, though more advanced methods exist. In the range of higher electron energies a pseudopotential calculation should be utilized because of the natural similarity of the conduction bands with free-electron parabolas.

The energy distribution of photoelectrons depends on both the initial- and final-state bands. For a first step in the interpretation of experimental spectra, so-called "band-mapping" methods have been developed. ${ }^{2}$ These methods only concern the position of the structures found in the spectra, relating them to the bands involved in the transition process, and they are only applicable in the model of direct transitions. The photoemission intensity remains undetermined.

This is the starting point of our work. Our aim is to calculate energy-distribution curves (EDC's) with regard to known band structures of the initial and final states. It necessarily involves the computation of the matrix elements of the phototransition between those states. We choose normal emission, because the interpretation of the results is simpler than in the off-normal case. Because of their importance in III-V compounds, the surface states have to be taken into account. To simplify the calculations, we do not apply time-reversed LEED (low-energy electron diffraction) states, which appear as final wave functions in the one-step model. ${ }^{4,5}$ This procedure is helpful in explaining the essential features of the EDC's.

To our knowledge these are the first calculated EDC's for GaAs including final states and matrix elements. In the following section we introduce our theoretical model before turning to the presentation of the results and the comparison with experimental data.

\section{METHOD OF CALCULATION}

\section{A. Photoemission}

The model is based on the "golden-rule" formulation of the photoemission according to Feibelman and Eastman and others. ${ }^{4}$ The intensity of the differential photocurrent is given by

$$
I \sim\left(E_{\mathrm{kin}}\right)^{1 / 2} \sum_{i, f}\left|\left\langle\Phi_{f}|D| \Phi_{i}\right\rangle\right|^{2} \delta\left(E_{f}-E_{i}-\hbar \omega\right),
$$

where the summation runs over all initial states $\Phi_{i}$ and final states $\Phi_{f}$ with energy $E_{i}$ and $E_{f}$, respectively, $E_{\text {kin }}$ is the kinetic energy of the photoelectrons, $D$ the dipole operator, and $\hbar \omega$ denotes the energy of the incident photons.

Many-particle effects influence the photoemission process in several ways. The photoelectron and the remaining hole are quasiparticles, which interact with the embedding electron gas. This effect is considered by introducing finite lifetimes in the single-particle calculations. However, we neglect any screening effects, i.e., we set the amplitude of the vector potential $A$ of the in- 
cident radiation equal to its vacuum value. For the dipole operator $D$, we write $D=\mathbf{A} \cdot \mathbf{p}$, where p denotes the momentum operator.

\section{B. Initial states}

Empirical tight-binding methods are known to give a good description of the valence bands of III-V semiconductor compounds. ${ }^{3}$ In our approach we take into account all interactions including third-nearest-neighbor atoms. Furthermore, we determine the transfer integrals according to the extended-Hückel-theory (EHT). ${ }^{6}$ It constitutes a relation between the elements of the overlap matrix $\underline{S}$ and those of the Hamiltonian matrix $\underline{H}$ :

$$
\begin{aligned}
& H_{\alpha \beta}=-\frac{1}{2} K_{\alpha \beta}\left(I_{\alpha}+I_{\beta}\right) S_{\alpha \beta}, \quad \alpha \neq \beta \\
& H_{\alpha \alpha}=-\widetilde{I}_{\alpha}-K_{\alpha \alpha} I_{\alpha}\left(S_{\alpha \alpha}-1\right) .
\end{aligned}
$$

$\alpha$ and $\beta$ enumerate the valence orbitals of each basis atom. ${ }^{7}$ The EHT has some advantages. First, there is a relatively small number of parameters to be fitted and this set does not depend on the number of neighboring atoms taken into account. We use 11 parameters for $\mathrm{GaAs}$, as there are four $I_{\alpha}$ and $\widetilde{I}_{\alpha}$ for each basic atomic orbital and three $K_{\alpha \beta}$, which are only labeled by the orbital symmetry, i.e., $K_{s s}, K_{s p}$, and $K_{p p}$. Second, it is not necessary to make any assumptions about the dependence of the transfer integrals on the binding length, as is usually done in tight-binding methods which neglect the overlap. Jansen and Sankey ${ }^{8}$ point out that the dependency is more similar to that of the overlap integrals than that of the often used $d^{-2}$ scaling law. ${ }^{9}$ The overlap integrals may be easily calculated for every interatomic distance, so that the dependence of the Hamiltonian matrix is given via (2.2) and (2.3). The EHT parameters are determined by fitting the bulk band structure to the one calculated by Chelikowsky and Cohen ${ }^{10}$ using the empirical pseudopotential method.

In order to determine the surface band structure of the (110) surface, we apply the Green's-function approach developed by López Sancho et al. ${ }^{11}$ within the ETBM framework. With this "layer-doubling" method we are able to calculate exactly the wave-vector- and layerresolved Green's functions of both surface and bulk layers. The Green's functions of any other crystal layer can be determined easily via transfer matrices. As our basis set consists of nonorthonormal atomic orbitals, we are forced to introduce a biorthonormal basis (see, for example, Ref. 12), which, however, does not explicitly enter the calculations. We distinguish it from the first basis set by a tilde.

\section{Final states and matrix elements}

To calculate the final states we use the empirical pseudopotential method (EPM) with the local potential of Cohen and Bergstresser. ${ }^{13}$ Instead of the usual determination of the energy of the wave functions depending on the wave vector $\mathbf{k}$, we use an approach which calculates the complex component $k_{\perp}$ of the wave vector perpendicular to the surface for a given energy and given wave-vector component parallel to the surface, $\mathbf{k}_{\| \cdot{ }^{14}}$ It is easy to introduce an optical potential into this procedure which describes the damping of the wave functions caused by many-body interactions. ${ }^{5,15}$ Its imaginary part $V_{i}$ determines the decay of the wave functions perpendicular to the surface. The energy dependence is parametrized as

$$
V_{i}(E)=\frac{c}{1+e^{-(E-\mu) / b}}+a .
$$

A similar dispersion is found for semiconducting layered crystals in calculations of the damping using the energyloss function $\operatorname{Im}\left(\epsilon^{-1}\right),{ }^{16}$ where $\epsilon$ is the dielectric constant. $V_{i}(E)$ increases for energies above the plasmon energy $\mu$, and thus the $k_{\perp}$ selection rule becomes weak.

In the calculation of the final states we take into account bulk bands, neglecting surface states and resonances. Surface states are not expected above the vacuum level as the band gaps are bridged over in the complex band structure because of the damping of the optical potential. Surface resonances are superpositions of the bulk states, thereby entering the calculation of the photoemission intensities even if the correct boundary condition at the surface is neglected. Thus, surface resonances are included, but they are not identified in this procedure.

It is easy to calculate the matrix elements of the dipole operator between these initial and final states analytical1y. ${ }^{17}$ They result as a sum over the Fourier transforms of the atomic wave functions, because the final-state wave functions in the pseudopotential approach are expanded into plane waves. A shortcoming of our model may be that very strong damped conduction bands cannot be included in the photoemission calculations, because the Fourier transformation would not converge if the decay of the atomic wave functions is smaller than that of the final state, the latter representing an exponential increase in the outgoing direction. The greatest decay taken into account is about $0.4 \AA^{-1}$. In this procedure we ignore the surface in extending the integration over the whole space. It may introduce an erroneous contribution to the matrix elements from the initial states located at the surface.

\section{Photocurrent}

After inserting the expressions for the initial and final states and calculating the matrix elements, one is able to evaluate the following formula for the intensity of the photocurrent, which is derived using Dirac's identity:

$$
I \sim\left(E_{\mathrm{kin}}\right)^{1 / 2} \operatorname{Im}\left[\sum_{i, j, f} M^{*}(f, i) \widetilde{G}_{i j}\left(E_{b}+i \eta\right) M(f, j)\right] .
$$

The sum runs over all final states $f ; i$ and $j$ denote layerand wave-vector-resolved Bloch sums with the binding energy $E_{b} . M(f, i)$ is the matrix element between initial state $i$ and final state $f$. $\widetilde{G}$ stands for the Green's function of the semi-infinite crystal in the biorthonormal representation resulting from the "layer-doubling" algorithm.

It is worth mentioning that our photoemission calculation uses two different Hamiltonians for deriving the en- 
ergy bands: a tight-binding Hamiltonian in the EHT approach for the initial states, and a pseudopotential Hamiltonian for the final states. A connection between these operators can be found by the Fourier transformation of the matrix elements of the pseudopotential Hamiltonian. Doing this yields the pseudopotential operator in the tight-binding notation. ${ }^{18}$ The matrix elements of the EHT Hamiltonian and those of the transformed operator show a very similar behavior in $\mathbf{k}$ space. A detailed analysis of this topic will be published elsewhere. The tight-binding approach cannot be used for the calculation of the final-state wave functions, which are rather extended in the energy range of the upper conduction bands. Therefore, as in the pseudopotential method, an expansion into plane waves is more suitable. Nevertheless, some tight-binding models ${ }^{19}$ take into account unoccupied atomic orbitals, resulting in a better description of the lower conduction bands which, however, are not relevant in the energy range discussed in this paper. In the valence-band regime the tight-binding method is more appropriate and, in addition, a surface is easily introduced within this formalism via a Green's-function algorithm.

\section{EXPERIMENT}

Photoemission spectroscopy with high angle and energy resolution is the most powerful experimental method of studying electronic band structures. To achieve high resolution we use a $180^{\circ}$ spherical analyzer mounted on a goniometer which is movable around two independent axes (improved Leybold-Heraeus three-dimensional angle-resolved electron spectrometer). The energy resolution chosen here was $100 \mathrm{meV}$ and the angle resolution better than $0.5^{\circ}$. The spectrometer is connected to the DORIS II storage ring at the Hamburg Synchrotron Radiation Laboratory (HASYLAB) using a normalincidence monochromator in the region $10 \leq \hbar \omega \leq 30 \mathrm{eV}$ and optional $\mathrm{He} \mathrm{I}$ radiation. The degree of polarization is between 80 and $99 \%$ depending on the photon energy.

The experiments were carried out on UHV-cleaved doped GaAs single crystals $\left(7.8 \times 10^{16}\right.$ carriers $\left./ \mathrm{cm}^{3}, \mathrm{Si}\right)$. The crystal orientation is controlled by conventional Laue and LEED techniques. The crystals are cleaved with a wedge working on both sides of a sawed groove parallel to the (110) surface supported by an adjustable anvil. This stress-free mounting yields the best-quality cleaves by application of minimum forces. In our system the sample is transferred after cleavage from a separate cleavage chamber to the photoemission chamber by magnetic transfer rods and then mounted in the focus of the synchrotron radiation on a manipulator.

The degrees of freedom, three of the manipulator and two of the electron analyzer, allow a very accurate final sample adjustment. By moving the sample, the geometry of the incident light relative to the sample normal and the mirror plane can be freely chosen. By moving the analyzer the $\bar{\Gamma}$ point of the chosen geometry can be found with high precision by studying the symmetry of the dispersing bands. As in the calculations, the experimental spectra are taken at an angle of $45^{\circ}$ for the incident light with the dominant component of the vector potential A lying in the mirror plane of the crystal.

\section{RESULTS AND DISCUSSIONS}

In the following subsections we first present the basic band structures and discuss the band mapping, before turning to the photoemission calculations.

\section{A. Theoretical band structures}

Our calculated valence bulk band structure is shown in Fig. 1. It is in good agreement with that calculated by Chelikowsky and Cohen ${ }^{10}$ neglecting relativistic effects, but the dispersion of the conduction bands is too wide. This shortcoming could be avoided using a greater number of EHT parameters, but we are only concerned with the valence bands. We specify the three valence bands in the energy range from $-7.0 \mathrm{eV}$ up to $0.0 \mathrm{eV}$ of the $\Sigma$ direction involved in the photoemission process by numbers. The uppermost initial state, no. 1, possesses $\Sigma_{2}$ symmetry, the initial state 2 is the band showing the $\Sigma_{\min }$, and the lowest one considered in the following discussion, no. 3 , refers to the band dispersing from $\Gamma_{15}$ to $X_{3}$.

The surface band structure calculated from the parameter fit with the bulk band structure is shown in Fig. 2.

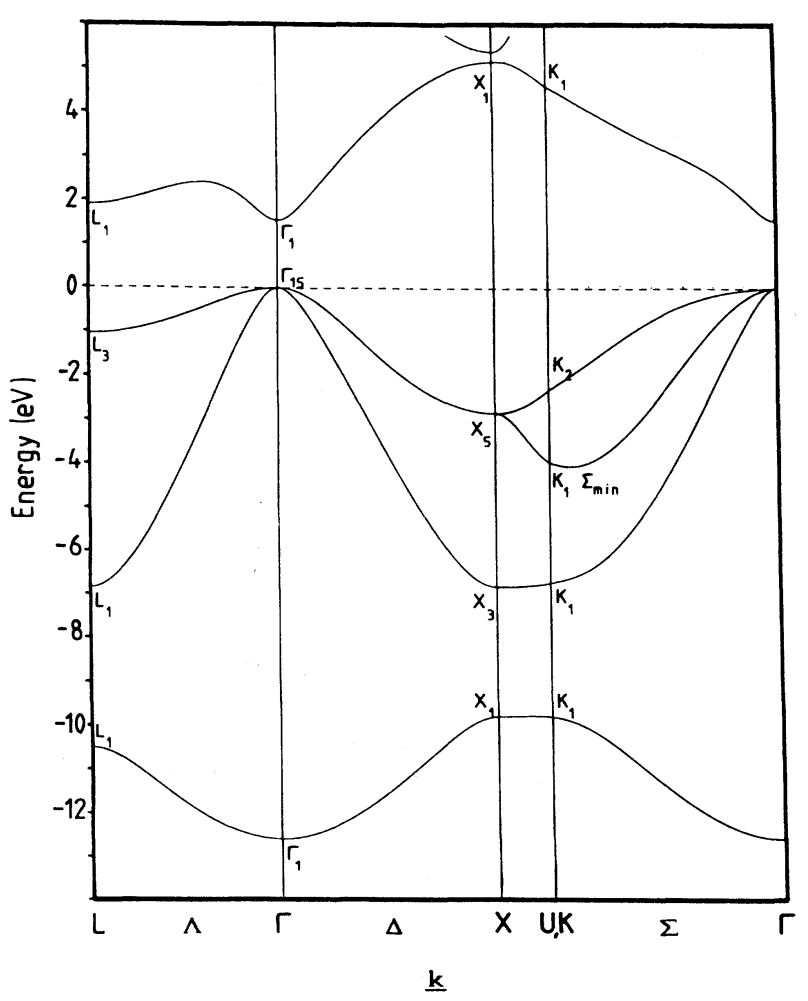

FIG. 1. Valence-band structure of bulk GaAs calculated in the tight-binding approach. The energy bands are shown along the high-symmetry lines of the bulk Brillouin zone. At the band edges the symmetry-group representation is specified. The zero of the energy refers to the valence-band maximum (VBM). 


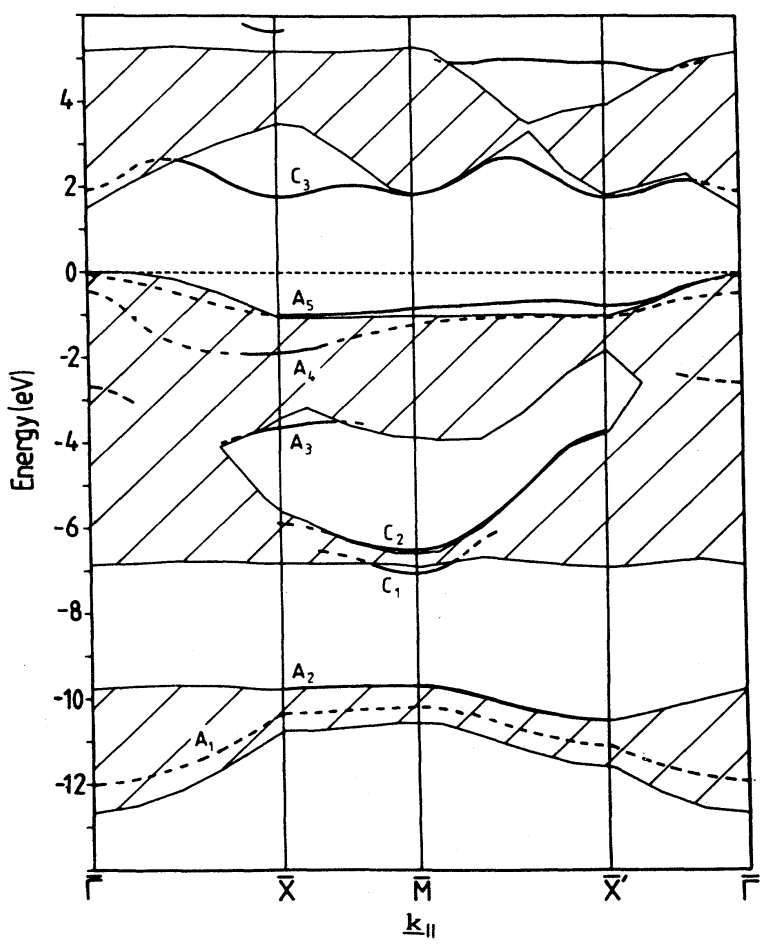

FIG. 2. Valence-band structure for the (110) surface of GaAs. The hatched areas mark the projected bulk band structure. Solid lines denote localized surface states, whereas dashed lines represent resonances. Anion-derived states are labeled with $A$ and cation-derived with $C$. The notation follows Ref. 20. The VBM is chosen as the energy zero.

There are several surface states and resonances, which we label the same way as Zhang and Cohen. ${ }^{20} \mathrm{We}$ choose the standard relaxation model of Duke et al., ${ }^{21}$ which is suggested and confirmed by LEED analysis. The agreement between our surface band structure and that of Zhang and Cohen is good.

Figure 3 shows the complex band structure of the symmetry line $\Sigma$ in the final-state energy regime calculated with a nonvanishing imaginary part of the optical potential [cf. Eq. (2.4)]. The small gaps appearing in a real band calculation are bridged over. Therefore the pronounced minima in the theoretical photoemission spectra, which would occur if the damping is considerably reduced, are removed. Depending on the energy the damping part of the optical potential decreases from $-0.3 \mathrm{eV}$ at small energies to $-1.8 \mathrm{eV}$ at higher energies, as assumed in Eq. (2.4) with our chosen parameters of $a=-0.3 \mathrm{eV}, b=2.5 \mathrm{eV}, c=-1.5 \mathrm{eV}$, and $\mu=17 \mathrm{eV}$. These values are found to be uncritical, because small changes in them leave the main features of the complex band structure as well as the calculated photoemission spectra unchanged. The main branches of the freeelectron parabolas can be clearly visualized. The final states of most of the possible transitions are expected to be associable with them as suggested earlier. ${ }^{2}$
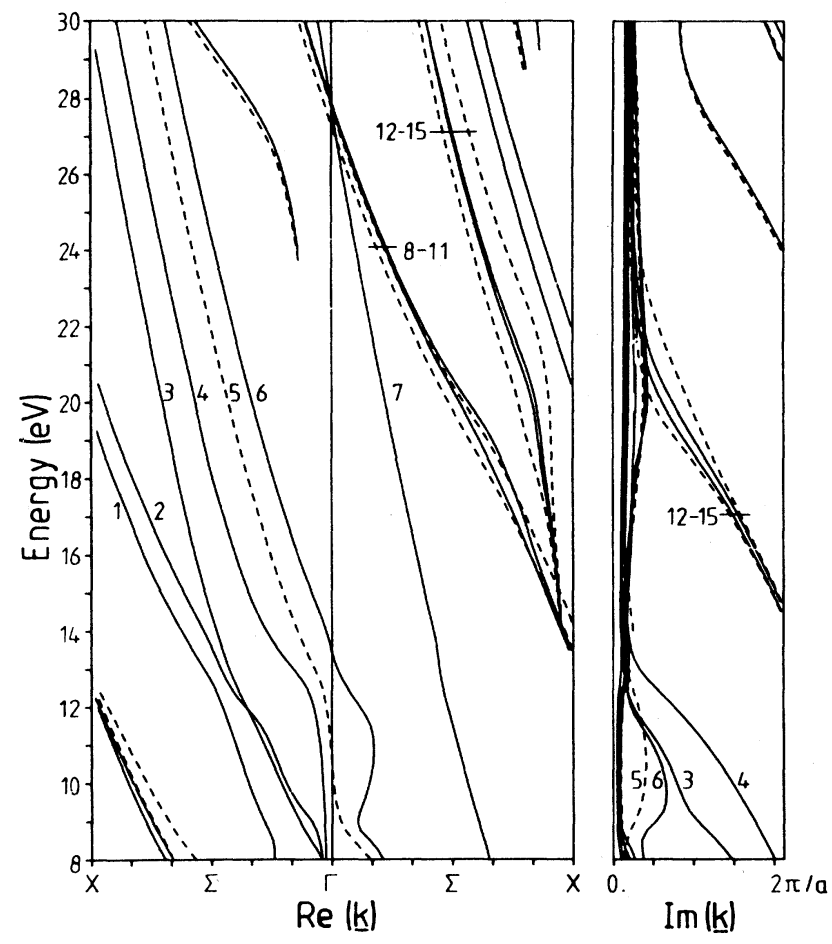

FIG. 3. Complex band structure of GaAs for the symmetry line $\Sigma$. The left inset shows the real part of $k_{1}$, the component of the wave vector perpendicular to the surface. The imaginary part is shown separately in the right inset. Only bands are given for which the damping is less than $2 \pi / a . \Sigma_{2}$ states are specified by dashed lines and $\Sigma_{1}$ states by solid lines. Bands labeled by numbers correspond to free-electron parabolas with following reciprocal-lattice vectors and their equivalent ones (Ref. 23): 1 and $2,(-1,-1,0) ; 3$ and $4,(-1,-1,-1) ; 5$ and $6,(-1,0,-1) ; 7$, $(-1,-1,-2) ; 8-11,(-2,-1,-1) ; 12-15,(-2,-1,-2)$.

\section{B. Structure plots}

After the determination of the energy-band structure of the initial and the final states, we are able to calculate the structure plots, which are useful for the interpretation of photoemission experiments, especially for normal emission. Assuming direct transitions, i.e., exact wavevector conservation, one determines the binding energies for which transitions are possible for a given excitation energy. In the resulting plots we omit the symmetryforbidden transitions because the vector potential of the incident radiation is chosen to lie in the mirror plane of the (110) surface corresponding to the experiment described above. These plots are shown in Fig. 4. The result for initial state 1 is not shown. Because of its $\Sigma_{2}$ symmetry it does not couple to the outgoing wave, which itself possesses $\Sigma_{1}$ symmetry in normal emission. It can be seen that most of the peaks (dots and circles in Fig. 4) from the experimental spectra (compare also Fig. 7) lie close to the theoretical direct transitions. Some of them also coincide with transitions from maxima of the $\mathbf{k}_{\|}$resolved density of states (DOS), i.e., $\Gamma_{15}, X_{5}, \Sigma_{\text {min }}$, and $X_{3}$. The peaks near the valence-band maximum (VBM) 


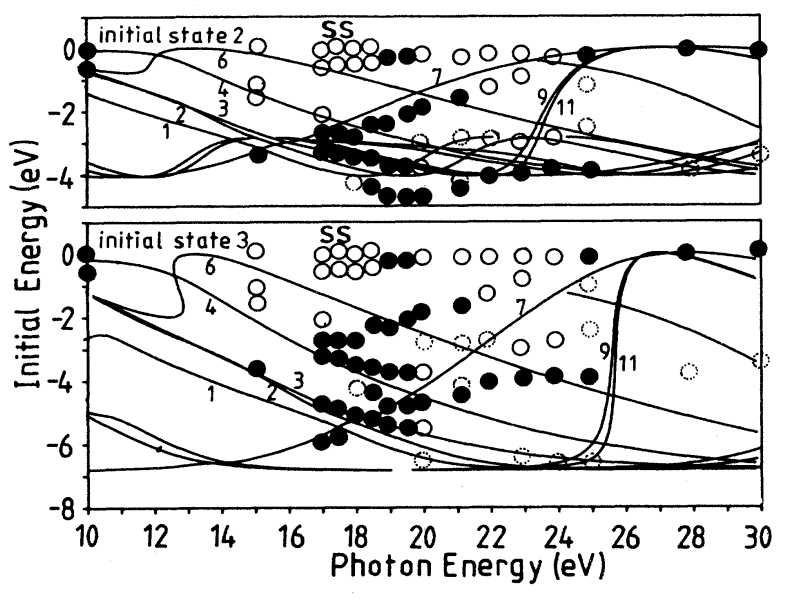

FIG. 4. Structure plots for $\Sigma_{1}$-symmetry initial states of the $\Sigma$ direction between -6.8 and $0.0 \mathrm{eV}$ labeled from the top of valence band to lower energies. Only symmetry-allowed transitions are shown. Solid dots specify pronounced peaks, open dots represent weaker structures. Very weak structures are marked with pointed circles. The zero of the initial energy is the VBM. The final bands are labeled as in Fig. 3. Dots arising from the dangling-bond surface state $A_{5}$ are denoted SS.

dispersing from about $-0.8 \mathrm{eV}$ at a photon energy of 17 $\mathrm{eV}$ up to the VBM at about $25 \mathrm{eV}$ excitation energy can be attributed to a transition from initial state 1 to final band 7, which is allowed only with a nonvanishing amount of $s$-polarized light. It can be explained by incomplete polarization and uncertainties of adjusting the plane of incidence with respect to the vector potential in the experiment. The nondispersive structure near the VBM, marked with SS in Fig. 4, arises from the surface state $A_{5}$ (compare Fig. 2). We establish that all bulkderived peaks can be associated with direct transitions within the possible error of the theoretical band structures.

\section{Photoemission spectra}

In Fig. 5 we present our calculated photoemission spectra. As in the experimental data, the main component of the polarization vector of the incident radiation lies in the mirror plane ( $p$ polarization) at an angle of $45^{\circ}$ to the surface normal. The photons are chosen to impinge on the surface in direction of the As dangling bonds. The imaginary part of the energy $\eta$ included in the Green's-function algorithm is set equal to $0.1 \mathrm{eV}$ in the whole valence-band range.

We observe that some of the structures found in the calculated spectra disperse with photon energy and some do not. The latter ones may be associated either with the maxima of the $\mathbf{k}_{\|}$-resolved density of states in the sense of $k_{\perp}$-relaxed direct transitions (cf. Fig. 4), here along the symmetry line $\Sigma$, or with surface states. In our spectra the nondispersive peaks are mainly caused by direct transitions. Surface states are too weak to be observed or lie

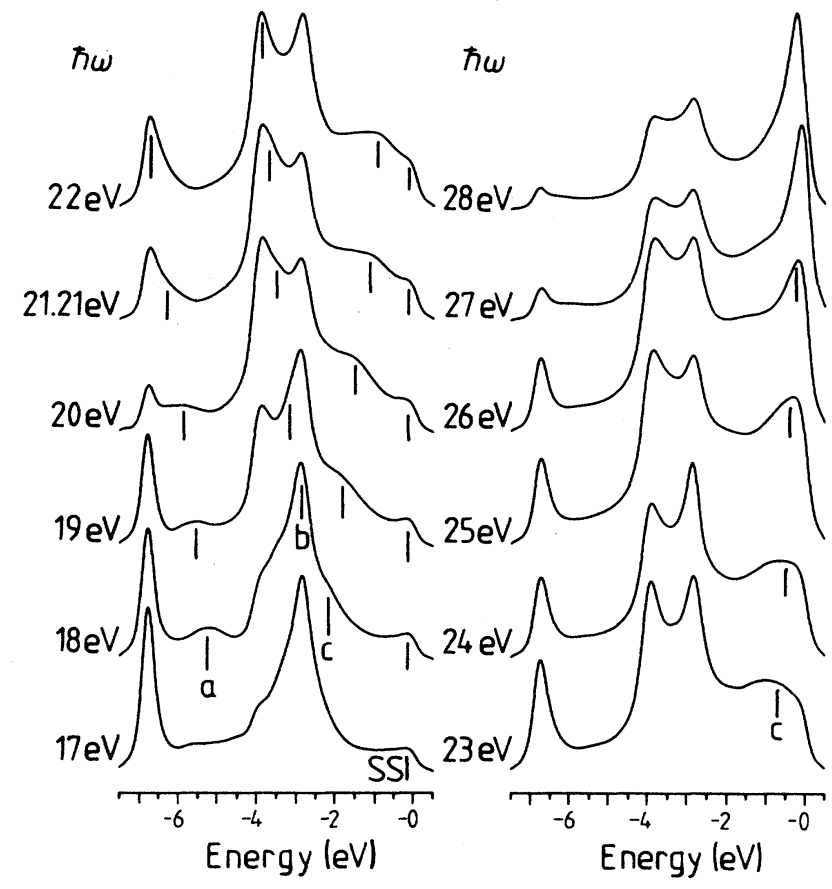

FIG. 5. Theoretical photoemission spectra for normal emission. The photon energy is shown at the low-energy edge of each spectrum. Dispersive structures, which are discussed in the text, are marked with lower case letters and tic marks. The surface state $A_{5}$ is denoted SS. The zero of the energy is the VBM, while that of the intensity corresponds approximately to the left end of a spectrum.

close to the band edges. In the latter case the separation between bulk and surface emission is difficult. This can be seen by the layer- and $k_{\|}$-resolved DOS for the point $\bar{\Gamma}$ (see Fig. 6). The surface-located peaks are faced with the overwhelming DOS of the valence-band maxima, i.e., $X_{3}$, $\Sigma_{\text {min }}, X_{5}$, and $\Gamma_{15}$, which are localized in the bulk layers. The relation of their intensities is similar to the one calculated by Chang. ${ }^{22}$ Only the dangling-bond state $A_{5}$ can be observed in the photoemission spectra when no strong transitions from the VBM appear, i.e., at photon energies between 16 and $23 \mathrm{eV}$ (labeled SS in Figs. 4 and 5). At higher photon energies the damping becomes so strong that the escape depth of the photoelectrons is very small and comparable with the interlayer distance. Therefore the spectra are given by the DOS of the first few crystal layers, except the pronounced peak near the valence-band maximum, which belongs to very strong transitions near $\Gamma$.

In Fig. 5 we observe three significant dispersive structures- $a, b$, and $c$-marked by bars. At a photon energy of $18 \mathrm{eV}$ a small peak, $a$, at about $-5.5 \mathrm{eV}$ can be observed. It disperses down to the $X_{3}$ peak, giving rise to the large maximum in the $22-\mathrm{eV}$ spectrum at $-6.8 \mathrm{eV}$. It is caused by emissions from initial state 3 to final bands 1-3. A second peak, $b$, can be traced by observing the intensity changes between the $\Sigma_{\min }$ and $X_{5}$ peaks. Up to 
$19 \mathrm{eV}$, the latter peak is stronger than the first, then the opposite is true until $22 \mathrm{eV}$. Subsequently, the $X_{5}$ peak rises again at high photon energies. This structure is caused by emissions from initial state 2 . The third dispersive peak, $c$, starts near the pronounced maximum at $-2.8 \mathrm{eV}$ of the $17-\mathrm{eV}$ spectrum. It moves to higher energies, reaching the valence-band maximum at about an excitation energy of $25 \mathrm{eV}$. This structure is due to a transition from initial state 2 to band 7 crossing $\Gamma$ at about $28 \mathrm{eV}$ in the complex band structure.

A detailed analysis of our spectra reveals that most transitions end on branches of the conduction-band structure that can be associated rather well with freeelectron-like parabolas.

In Fig. 7 the experimental spectra are shown for selected photon energies. The incident radiation coincides with that in the calculations. Comparing these spectra with the calculated ones, Fig. 5, we observe, in general, satisfactory agreement within the limits of our model. We now compare the spectra according to energy and intensity in more detail. For the $28-\mathrm{eV}$ spectrum we find good agreement between theory and experiment. In both

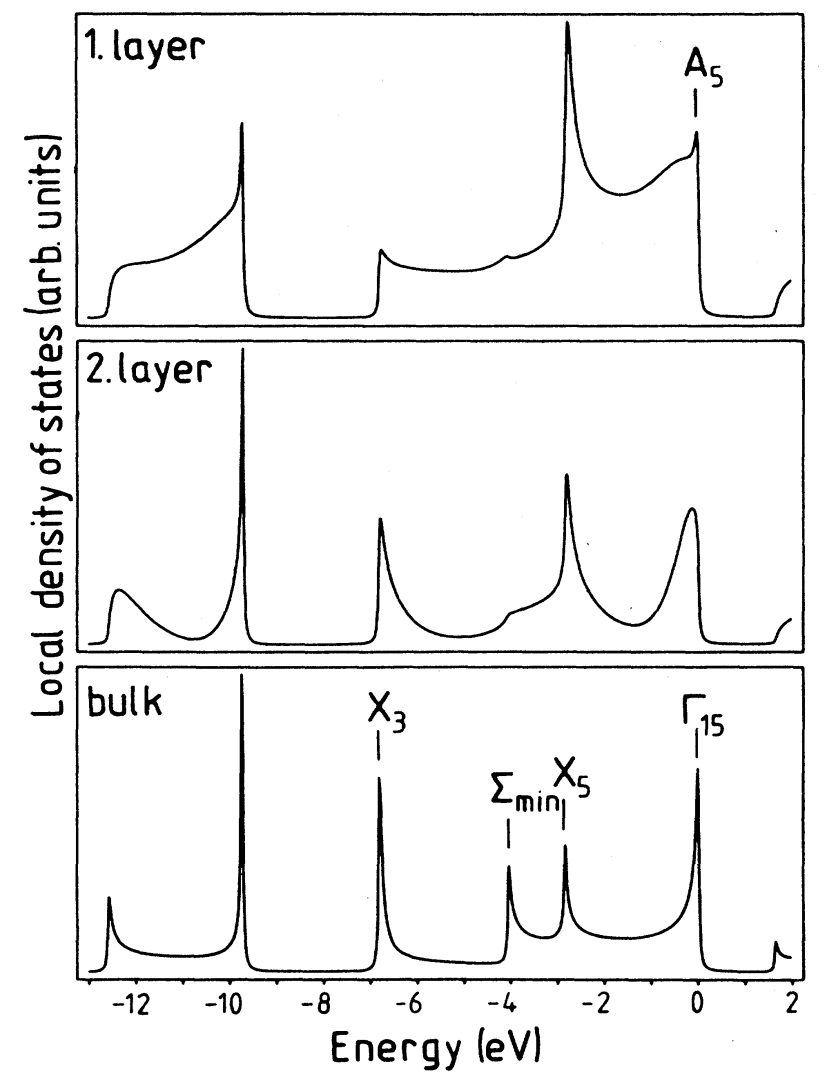

FIG. 6. Layer- and $\mathbf{k}_{\|}$-resolved density of states for the first, the second, and a bulk layer at $\bar{\Gamma}$. The main maxima are labeled with the group representation of the band edge, if they are bulk features, or with the name of the surface state they are attributed to. The zero of energy refers to the VBM; the broadening is $0.02 \mathrm{eV}$.
EDC's one peak near the VBM dominates, which is created by strong transitions near $\Gamma$ (cf. Figs. 3 and 4). Emissions from the $X_{3}, \Sigma_{\text {min }}$, and $X_{5}$ points are significantly reduced. In this range of photon energies the experimental peaks are broader than the theoretical ones.

This good correspondence between theory and experiment also holds for spectra taken at lower photon energies. See, e.g., peak $c$ in the experimental spectra, starting near the $X_{5}$ peak at a photon energy of $17 \mathrm{eV}$ and reaching the VBM at a photon energy of about $27 \mathrm{eV}$, which can be clearly identified with the theoretical structure $c$ (cf. Figs. 4 and 5). Exceptions seem to be emissions marked with $d$ and $e$ in Fig. 7. Peak $d$, corresponding to transitions from initial state 3 into the free-electron-like final band 7, disperses through the whole experimentally derived valence-band range and can be found only as a hint near the minimum at $-1.5 \mathrm{eV}$ for photon energies about $26 \mathrm{eV}$ in the theoretical spectra. This difference may be explained by the bulk-vacuum coupling, which is neglected in the calculations. Final state 7 is expected to couple very strong to the outgoing wave, because the main contribution of the wave function should come

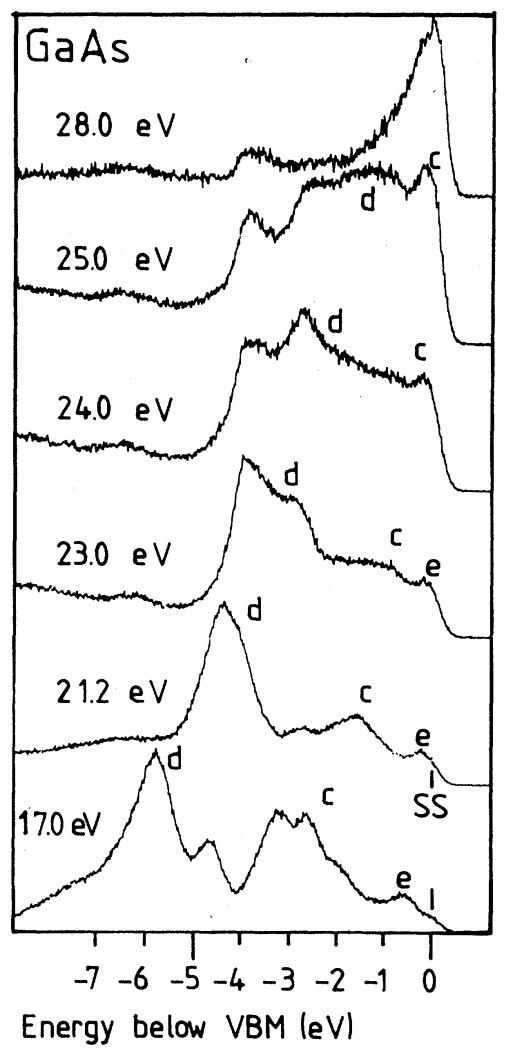

FIG. 7. Experimental energy distribution of photoelectrons for normal emission excited with $p$-polarized radiation. The photon energy is indicated at the left edge of each spectrum. Structures discussed in the text are denoted by lower case letters, except the surface state $A_{5}$, which is marked by SS and tic marks. The energy zero is the VBM. 
from Fourier components of wave vectors aligned to the surface normal. Therefore, this shortcoming of the theory may be resolved, if the escape of the photoelectrons is explicitly taken into account, resulting in an emphasis of the transitions mentioned above. Peak $e$ in the experimental spectra corresponds to transitions from initial state 1 into final band 7, which for reasons discussed in Sec. IV B are strictly not allowed. It is not considered for the theoretical spectra.

In general, the spectral range between the $X_{5}$ peak and the VBM is good reproduced by the calculations; see, e.g., the 23-eV spectra. Only the $X_{5}$ and $X_{3}$ peaks are a little more pronounced by the theory, as can be also seen for lower photon energies. It should be mentioned that the intensities from critical points $X_{3}, \Sigma_{\text {min }}$, and $X_{5}$ seem to be generally overestimated in the theoretical spectra.

We have also studied the polarization dependence of some spectra. We changed the geometry of incidence by rotating the sample around the polar axis by $180^{\circ}$. For instance, at $23 \mathrm{eV}$ photon energy the shoulder at $-2.8 \mathrm{eV}$ rises and dominates the experimental EDC. This is reproduced by our calculations, but the change is smaller than in experiment. There is also similar agreement for spectra at other photon energies.

Thus, it is established that the four nondispersive and the three dispersive structures can be traced in agreement with the experiment.

\section{CONCLUSIONS}

The photoemission spectra of $\mathrm{GaAs}(110)$ for emission normal to the surface are calculated within a model which includes final states as well as matrix elements in order to interpret experimental valence-band spectra taken at low photon energies with synchrotron radiation. These are expected to exhibit significant modulations in intensity due to the detailed structure of the final states.
We find that the positions of all peaks in the experimental spectra can be explained as direct bulk transitions except the peak near the VBM, which is explained by a surface state. Structure plots show that, within the limits of the theoretical model and the evaluation of the experimental data, the experimentally observed peaks correspond very well with the theoretically predicted direct transitions. In comparing the experimental energy-distribution curves with those determined by our theoretical model, we also find an overall satisfactory agreement. The characteristic experimental features concerning the variation of the relative intensities with photon energy are fairly well reproduced in the calculations. In the strongdamping limit, i.e., at high photon energies, we observe much better agreement than at lower excitation energies. The dangling-bond state $A_{5}$ can be detected in that photon-energy range where no direct transitions from the valence-band maximum occur. Other surface states play no significant role, because their energies almost coincide with those of the maxima of the bulk density of states. In the next stage of our work the escape of the photoelectrons from the solid into the vacuum region will be explicitly taken into account in order to investigate additionally the effect of bulk-vacuum coupling on the spectra, which can be considered to be responsible for the remaining differences between our calculations and the experiment.

\section{ACKNOWLEDGMENTS}

We gratefully acknowledge the support by the Rechenzentrum der Universität Kiel, the Konrad-Zuse-Zentrum für Informationstechnik Berlin, as well as the Deutsche Forschungsgemeinschaft. The experimental work was supported by the German Federal Minister of Research and Technology (BMFT) under Contract No. 05-301AAI.
${ }^{1}$ P. E. Gregory and W. E. Spicer, Phys. Rev. B 13, 725 (1976); A. Huijser, J. van Laar, and T. L. van Rooy, Phys. Lett. 65A, 337 (1978).

${ }^{2}$ G. P. Williams, F. Cerrina, G. J. Lapeyre, J. R. Anderson, R. J. Smith, and J. Hermanson, Phys. Rev. B 34, 5548 (1986), and references therein.

${ }^{3}$ C. Mailhiot, C. B. Duke, and D. J. Chadi, Phys. Rev. B 31, 2213 (1985); J. Pollmann, R. Kalla, P. Krüger, A. Mazur, and G. Wolfgarten, Appl. Phys. A 41, 21 (1986).

${ }^{4}$ P. J. Feibelman and D. E. Eastman, Phys. Rev. B 10, 4932 (1974); for references, see, e.g., A. Liebsch, in Festkörperprobleme XIX, edited by J. Treusch (Vieweg, Braunschweig, 1979).

5 J. B. Pendry, Surf. Sci. 57, 679 (1976).

${ }^{6}$ R. Hoffmann, J. Chem. Phys. 39, 1397 (1963).

${ }^{7}$ E. Clementi and C. Roetti, At. Data Nucl. Data Tables 14, 177 (1974).

${ }^{8}$ R. W. Jansen and O. F. Sankey, Phys. Rev. B 36, 6520 (1987).

${ }^{9}$ W. A. Harrison, Electronic Structure and the Properties of Solids (Freeman, San Francisco, 1980).

10J. R. Chelikowsky and M. L. Cohen, Phys. Rev. B 14, 556
(1976).

${ }^{11}$ M. P. López Sancho, J. M. López Sancho, and J. Rubio, J. Phys. F 15, 851 (1985).

${ }^{12}$ L. E. Ballentine and M. Kolâr, J. Phys. C 19, 981 (1986).

${ }^{13}$ M. L. Cohen and T. K. Bergstresser, Phys. Rev. 141, 789 (1966).

14J. B. Pendry, J. Phys. C 2, 2273 (1969).

15J. K. Grepstad, B. Slagsvold, and I. Bartoš, J. Phys. F 12, 1679 (1982).

${ }^{16}$ A. E. Smith, Phys. Status Solidi B 123, 619 (1984).

17J. W. Gadzuk, Phys. Rev. B 10, 5030 (1974).

18J. Q. Bartling and R. A. Craig, Phys. Rev. B 3, 3170 (1971).

${ }^{19}$ P. Vogl, H. P. Hjalmarson, and J. D. Dow, J. Phys. Chem. Solids 44, 365 (1983).

${ }^{20}$ S. B. Zhang and M. L. Cohen, Surf. Sci. 172, 754 (1986).

${ }^{21}$ C. B. Duke, S. L. Richardson, A. Paton, and A. Kahn, Surf. Sci. 127, L135 (1983).

${ }^{22}$ Y.-C. Chang, Phys. Rev. B 26, 4400 (1982).

${ }^{23}$ C. J. Bradley and A. P. Cracknell, The Mathematical Theory of Symmetry in Solids (Clarendon, Oxford, 1972). 